\title{
Peroxidase-Like Catalytic Activity of Anion-Exchange Resins Modified with Metal-Porphyrins in Oxidative Reaction of Hetrocyclic Amines with Hydrogen Peroxide
}

\author{
Masaki Mifune, ${ }^{*, a}$ Daiki Hino, ${ }^{a}$ Hikaru Sugita,,${ }^{a}$ Akimasa Iwado, ${ }^{a}$ Youji Kitamura, ${ }^{a}$ \\ Noriko Мотонаsнi, ${ }^{b}$ Ikuko TsuкAmoto, ${ }^{c}$ and Yutaka SaIto ${ }^{a}$ \\ ${ }^{a}$ Faculty of Pharmaceutical Sciences, Okayama University; Tsushima-Naka, Okayama 700-8530, Japan: ${ }^{b}$ Kobe \\ Pharmaceutical University; Motoyama-kita-machi, Higashinada-ku, Kobe 658-8558, Japan: and ${ }^{c}$ Faculty of Medicine, \\ Kagawa University; Ikenobe, Miki-Cho, Kagawa 761-0793, Japan. \\ Received April 16, 2005; accepted May 19, 2005; published online May 23, 2005
}

To elucidate the peroxidase (POD)-like catalytic activity of anion-exchange resins modified with metaltetrakis(sulfophenyl)porphine (M-TSPP $r$ s), an oxidative reaction of seven mutagenic heterocyclic amines (HCAs) with hydrogen peroxide, which reaction is catalyzed by horse radish POD, was investigated in the presence of MTSPP $_{r}$ s. Among six M-TSPP $r$ tested, Mn- and Fe-TSPP $r$ were found to have a relatively strong POD-like activity for HCAs, in particular for a typical HCA, 2-amino-3-methyl-imidazo[4,5-f lquinoline (IQ). The optimal condition for the POD-like activity was selected using Fe- and Mn-TSPP $\mathrm{r}_{\mathrm{r}}$. For evaluation of an oxidation product of IQ produced in the presence of Fe-TSPP , the absorption, NMR and FAB-mass spectra thereof were compared with those of an oxidation product of IQ produced by horse radish POD or a chemical oxidizing agent, sodium

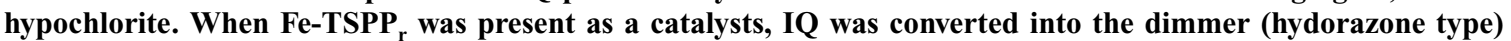
which has no mutagenic activity in $u m u$-test. It was revealed that Fe- and Mn-TSPP $s$ exhibit a POD-like catalytic activity in oxidative reaction of HCAs with hydrogen peroxide.

Key words hetrocyclic amine; peroxidase-like activity; metal-porphyrin; oxidative reaction

Heterocyclic amines (HCAs, Fig. 1) are mutagenic factors that are predominantly ingested as pyrolysis products of meats and fishes. ${ }^{1)}$ To adsorb the HCAs on ligands, a cellulose coupled with a $\mathrm{Cu}$-phthalocyanine derivative $(\mathrm{Cu}-\mathrm{PC}$, a related compound metal-porphyrin), so-called "blue cotton," was developed, ${ }^{2,3)}$ which was reported to be beneficial for callbacks and determinations of HCAs in environmental waters $^{2)}$ and air-born particules. ${ }^{4)}$ The adsorption of HCAs on the blue cotton is caused by hydrophobic $\pi-\pi$ interaction between HCAs and $\mathrm{Cu}-\mathrm{PC}$ coupled with cotton fiber. Peroxi-
10<smiles>Cc1c(N)[nH]c2c1CCCN1CCCCC21</smiles>

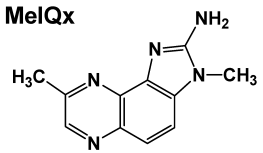<smiles>Cc1nc(N)cc2[nH]c3ccccc3c12</smiles>

GluP-1

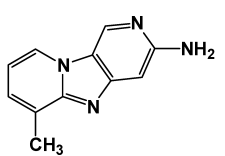

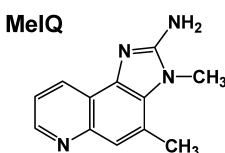

TrpP-1 $\mathrm{H}_{3} \mathrm{C}$

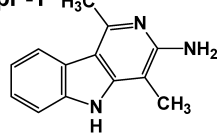

GluP-2

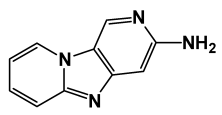

Fig. 1. Structures of HCAs Tested dase (POD), of which active cite is Fe-protoporphyrin, was reported to catalyze oxidative reactions of a wide range of mutagens and carcinogens including HCAs, thereby decreasing the mutagenicity and carcinogenicity. ${ }^{5,6)}$ In the above cases, metal-porphyrin derivatives serve as an adsorbent of HCAs or a catalysis of oxidative reaction of mutagens.

On the other hand, we previously reported that some anion-exchangers modified with metal-tetrakis(sulfophenyl)porphines $\left(\mathrm{M}-\mathrm{TSPP}_{\mathrm{r}}\right.$ ) exhibited a peroxidase (POD)-like activity and was applicable to the determination of hydrogen peroxide as a solid-mimesis of horse radish POD. ${ }^{7,8}$ In the present paper, we investigated the POD-like activity of M$\mathrm{TSPP}_{\mathrm{r}} \mathrm{s}$ in the oxidation of HCAs to elucidate the usefulness of M-TSPP $\mathrm{s}$ as a POD-like catalyst in the oxidation of HCAs. We revealed that some M-TSPP $r$ s exhibit a similar POD-like activity for HCAs to horse radish POD. In particular, Fe- and Mn-TSPP ${ }_{r}$ c catalyze the oxidation of 2-amino-3methyl-imidazo[4,5- $f$ ] quinoline (IQ) with hydrogen peroxide. The resulting IQ-dimmer has no mutagenicity in umutest. We elucidated that M-TSPP ${ }_{r}$ can decrease mutagenicity of HCAs in vitro through its POD-like catalytic activity.

\section{Experimental}

Materials Tetrakis(sulfophenyl)porphine $\left(\mathrm{H}_{2}\right.$-TSPP) was purchased from Wako Junyaku Co. Ltd. (Osaka, Japan). Mutagenic HCAs (see Fig. 1), IQ, MeIQ, MeIQx, TrpP-1 and TrpP-2, were purchased from Wako Junyaku Co. Ltd. GluP-1 and GluP-2 (Chemsyn Science Laboratories, Lenaxa, Kansas, U.S.A.) were kindly donated from Prof. Hikoya Hayatsu (Shujitsu University). They were used without further purification. An anion-exchange resin, Amberlite IRA 900 (24-42 mesh) in nitrate form, was a manufacture of Organo Chemical Co. Ltd. (Tokyo, Japan). Other reagents were of analytical or reagent grade. $u m u$-Test kit was purchased from Japan Immunoresearch Laboratories Co. Ltd. (Takasaki, Japan).

Apparatus Absorption (UV/V) spectra and absorbances (abbr. " $A$ ") were measured on a Shimadzu UV-180 or a JASCO V-570 spectrophotometer with $10 \mathrm{~mm}$ quartz cells. HPLC system was constructed with a Shimadzu pump (LC-6A), a Shimadzu photodiode array detector (SPD-M6A) and a 


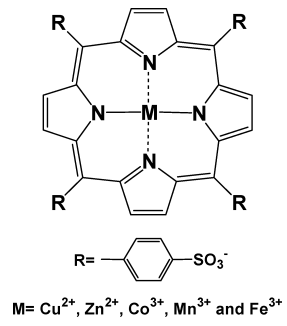

Fig. 2. Structure of M-TSPP

ODS column (Cosmosil packed column 5C18-AR, $\phi 4.6 \times 150 \mathrm{~mm}$, Nacalai Tesque, Kyoto, Japan). FAB-mass and ${ }^{1} \mathrm{H}-\mathrm{NMR}$ spectra were measured on a VG 70-SE (Manchester, U.K.) and a Varian VXR-500 (Polo Alto, CA, U.S.A.) instruments, respectively.

Modification of Amberlite IRA 900 with Metal-Porphyrins Metaltetrakis(sulfophenyl)porphine (M-TSPP, $\mathrm{M}=\mathrm{Cu}^{2+}, \mathrm{Zn}^{2+}, \mathrm{Co}^{3+}, \mathrm{Mn}^{3+}, \mathrm{Fe}^{3+}$, see Fig. 2) were prepared as described in the literatures. ${ }^{9,10)}$ An anion-exchange resin modified with M-TSPP $\left(\mathrm{M}-\mathrm{TSPP}_{\mathrm{r}}\right)$ was prepared by incubating $4.0 \mathrm{~g}$ of dry Amberlite IRA 900 with a solution of M-TSPP $(100 \mu \mathrm{mol}$ in $200 \mathrm{ml})$ in a mixture of acetone and water $(1: 1)$ for $8 \mathrm{~h} .{ }^{11)}$ In all cases, MTSPPs were adsorbed completely on IRA 900 and not detected at all in the solution after incubation. No elution of M-TSPP was observed when the M$\mathrm{TSPP}_{\mathrm{r}}$ was shaken with water and/or methanol. The M-TSPP $\mathrm{s}_{\mathrm{s}}$ could be preserved in dark stably for at least one year at room temperature.

Recommended Procedure for Estimation of POD-Like Activity for Mutagens Fifty milligrams of M-TSPP ${ }_{\mathrm{r}}$ was added into an aqueous mixture of a HCA solution $(0.25-0.50 \mathrm{mmol} / 1,1.0 \mathrm{ml})$, a hydrogen peroxide solution $(10 \mathrm{mmol} / 1,1.0 \mathrm{ml})$ and a $\mathrm{pH} 7.0$ borate buffer solution $(8.0 \mathrm{ml})$, and the mixture was incubated at $35^{\circ} \mathrm{C}$ for $2 \mathrm{~h}$. The M-TSPP was filtered off and the UV/V spectrum of the supernatant was measured. The HCA and its oxidation product adsorbed on the M-TSPP were extracted with $10.0 \mathrm{ml}$ of methanol for $3 \mathrm{~h}$. The M-TSPP ${ }_{\mathrm{r}}$ was filtered off, and the methanol-eluate was subjected to analyses such as FAB-mass spectroscopy and separation by HPLC.

The oxidation product in the methanol-eluate was separated from HCA by column chromatography (packed with octadecylsilyl-silica-gels, ODS) before measurement of ${ }^{1} \mathrm{H}-\mathrm{NMR}$ spectra.

Separation of POD-Catalyzed Oxidation Product of IQ One milliliter of horse-radish POD solution $(50 \mathrm{unit} / \mathrm{ml})$ was added into a mixture of IQ $(0.25 \mathrm{mmol} / 1,1.0 \mathrm{ml})$, hydrogen peroxide $(10 \mathrm{mmol} / 1,1.0 \mathrm{ml})$ and buffer $(\mathrm{pH} 7.0,8.0 \mathrm{ml})$ solutions. The mixture was incubated at $35^{\circ} \mathrm{C}$ for $30 \mathrm{~min}$, and the resulting precipitates were filtered off, washed with water and dried.

Oxidation of IQ with Sodium Hypochlorite and Separation of Product Five milliliters of sodium hypochlorite solution ( $c a$. 10\%) was added into a solution of IQ $(0.25 \mathrm{~mm}, 50 \mathrm{ml})$. The mixture was kept at $35^{\circ} \mathrm{C}$ for $1 \mathrm{~h}$, and the resulting precipitates were filtered off, washed with water and dried. The precipitates were recrystallized from methanol to obtain the pure oxidation product of IQ (IQ dimmer).

\section{Result and Discussion}

UV/V Spectra of Reaction Mixtures after Incubation with M-TSPP $\mathbf{r}$ S Typical UV/V spectra are shown in Fig. 3. When an aqueous mixture of IQ and hydrogen peroxide solutions was incubated with Fe-TSPP ${ }_{r}$, the absorbance at $263 \mathrm{~nm}$ resulting from IQ markedly decreased. This decrease could be caused by not only oxidation of IQ but also adsorption of IQ on IRA 900 and/or on Fe-TSPP on IRA 900 through the $\pi-\pi$ electron interaction by analogy with the cases of blue resin. ${ }^{12,13)}$ The observed $A$ values at $263 \mathrm{~nm}$ of the mixtures after incubation are summarized in Fig. 4 as $A_{\text {aq. }}$. As can be seen from Fig. 4, IRA 900 adsorbs IQ by half of the amount added. $\mathrm{H}_{2}-, \mathrm{Cu}-, \mathrm{Zn}-$ and $\mathrm{Co}-\mathrm{TSPP}_{\mathrm{r}} \mathrm{s}$ adsorb IQ at the similar level to IRA 900 . It is of interest that $A_{\text {aq }}$ value in the case of $\mathrm{Mn}$ - and Fe-TSPP $\mathrm{r}$ is much smaller than that in IRA 900. This result indicates that $\mathrm{Mn}$ - and Fe-TSPP $\mathrm{s}$ adsorb and/or oxidize more IQ than IRA 900. However, no extra UV/V

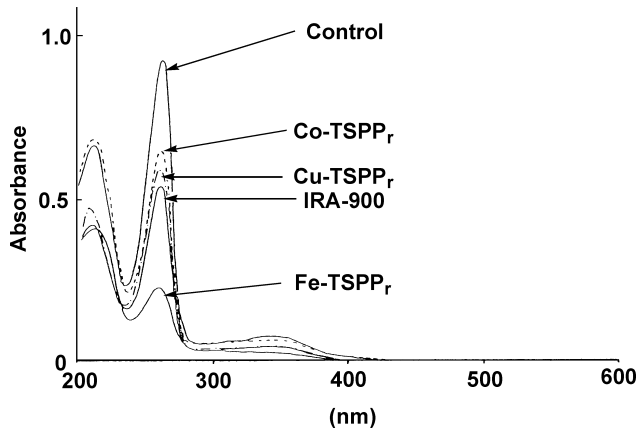

Fig. 3. Absorption (UV/V) Spectra of the Reaction Mixtures Containing IQ after Incubation

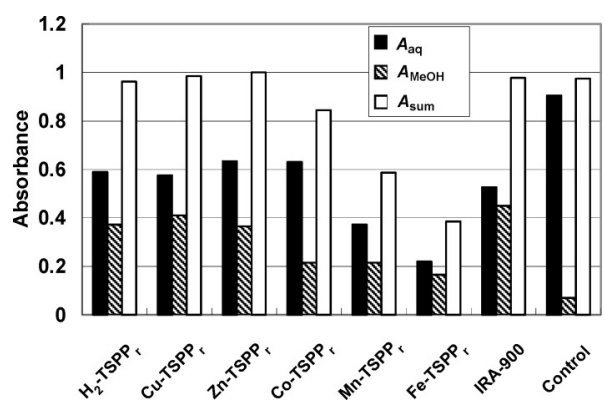

Fig. 4. Absorbances at $263 \mathrm{~nm}$ of Reaction Mixture Containing IQ $\left(A_{\mathrm{aq}}\right)$, Methanol-Eluates $\left(A_{\mathrm{MeOH}}\right)$ and Sums of $A_{\text {aq }}$ and $A_{\mathrm{MeOH}}$

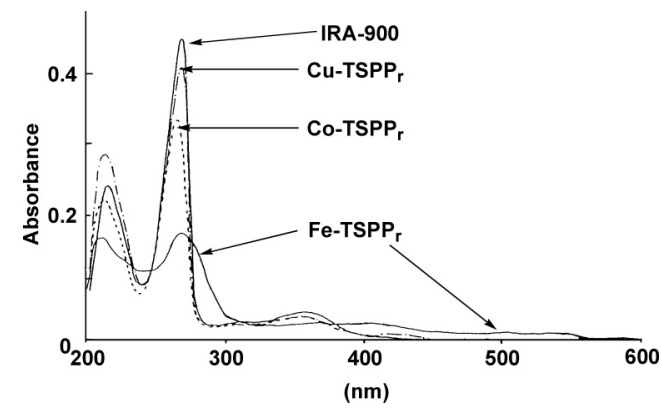

Fig. 5. Absorption (UV/V) Spectra of the Methanol-Eluates with Methanol

bands other than the band due to IQ were observed in all the cases as demonstrated by Fig. 3. This result suggests that any oxidation products of IQ do not exist in the aqueous phase, which was confirmed by HPLC. Accordingly, if M-TSPP $\mathrm{r}$ could catalyze the oxidative reaction of IQ, the products should be adsorbed by M-TSPP $\mathrm{r}$. Thus, we examined the adsorbates on respective M-TSPP $\mathrm{r}$.

Methanol-Eluates from M-TSPP $\mathbf{r}$ s To examine the adsorbates on the resins, we compared UV/V spectra of methanol-eluates obtained from reactions with various $\mathrm{M}$ $\mathrm{TSPP}_{\mathrm{r}} \mathrm{s}$ and IRA 900. The typical UV/V spectra are shown in Fig. 5. As can be seen from Fig. 5, the adsorbates on the M$\mathrm{TSPP}_{\mathrm{r}}$ were eluted with methanol. In the cases of $\mathrm{H}_{2-}, \mathrm{Cu}-$, $\mathrm{Zn}$ - and Co-TSPP $\mathrm{s}$, no extra bands other than the UV/V bands due to IQ were observed, similarly to the case of IRA 900 alone. In these cases, the sums of $A_{\text {aq }}$ and absorbance of methanol-eluate $\left(A_{\mathrm{MeOH}}\right)$ are close to one another (Fig. 4), suggesting that IQ added remained unchanged. This indicates that IQ is not oxidized by hydrogen peroxide in these 
Table 1. Absorbances of Reaction Mixtures $\left(A_{\mathrm{aq}}\right)$ and Methanol-Eluates $\left(A_{\mathrm{MeOH}}\right)$, and Sums $\left(A_{\mathrm{sum}}\right)$ of $A_{\mathrm{aq}}$ and $A_{\mathrm{MeOH}}$ at Absorption Maximum $\left(\lambda_{\max }\right)$ of Each HCA

\begin{tabular}{|c|c|c|c|c|c|c|c|}
\hline $\begin{array}{l}\text { Mutagen } \\
\lambda_{\max }(\mathrm{nm})\end{array}$ & $\begin{array}{c}\text { IQ } \\
263\end{array}$ & $\begin{array}{c}\text { MeIQ } \\
263\end{array}$ & $\begin{array}{l}\text { MeIQx } \\
263\end{array}$ & $\begin{array}{c}\text { TrpP-1 } \\
262\end{array}$ & $\begin{array}{c}\text { TrpP-2 } \\
262\end{array}$ & $\begin{array}{c}\text { GluP-1 } \\
243\end{array}$ & $\begin{array}{c}\text { GluP-2 } \\
243\end{array}$ \\
\hline $\begin{array}{c}\mathrm{Fe}-\mathrm{TSPP}_{\mathrm{r}} \\
A_{\mathrm{aq}} \\
A_{\mathrm{MeOH}} \\
A_{\text {sum }}\end{array}$ & $\begin{array}{l}0.220 \\
0.165 \\
0.385\end{array}$ & $\begin{array}{l}0.408 \\
0.354 \\
0.768\end{array}$ & $\begin{array}{l}0.660 \\
0.230 \\
0.890\end{array}$ & $\begin{array}{l}0.630 \\
0.700 \\
1.330\end{array}$ & $\begin{array}{l}0.515 \\
0.520 \\
1.035\end{array}$ & $\begin{array}{l}0.200 \\
0.145 \\
0.345\end{array}$ & $\begin{array}{l}0.296 \\
0.138 \\
0.434\end{array}$ \\
\hline $\begin{array}{c}\mathrm{Mn}-\mathrm{TSPP}_{\mathrm{r}} \\
A_{\mathrm{aq}} \\
A_{\mathrm{MeOH}} \\
A_{\text {sum }}\end{array}$ & $\begin{array}{l}0.372 \\
0.215 \\
0.587\end{array}$ & $\begin{array}{l}0.324 \\
0.286 \\
0.610\end{array}$ & $\begin{array}{l}0.510 \\
0.140 \\
0.650\end{array}$ & $\begin{array}{l}0.738 \\
0.450 \\
1.188\end{array}$ & $\begin{array}{l}0.530 \\
0.485 \\
1.015\end{array}$ & $\begin{array}{l}0.230 \\
0.100 \\
0.330\end{array}$ & $\begin{array}{l}0.290 \\
0.120 \\
0.410\end{array}$ \\
\hline$A_{\text {control }}^{a}{ }^{a}$ & 0.905 & 0.928 & 1.300 & 1.600 & 1.310 & 0.430 & 0.610 \\
\hline
\end{tabular}

a) Absorbances of each reaction mixture before addition of Fe- or Mn-TSPP

cases. However, in the cases of Mn- and Fe-TSPP $\mathrm{r}$, the methanol-eluates were colored reddish, and new weak bands around $500 \mathrm{~nm}$ were observed. As described below, the eluate containing only IQ and the colored compound which was revealed to be an oxidation product of IQ by the POD like activity of Mn- and Fe-TSPP $\mathrm{r}$. In addition, as can be seen from Fig. 4, the sums $\left(A_{\text {sum }}\right)$ of $A_{\text {aq }}$ and $A_{\mathrm{MeOH}}$ were much smaller than that in the case of IRA 900, which means that a part of IQ used was changed. Accordingly, we estimated that $\mathrm{Mn}$ - and Fe-TSPP $\mathrm{r}$ s catalyzed an oxidative reaction of IQ in the presence of hydrogen peroxide. We then examined the catalytic activity of Mn- and $\mathrm{Fe}-\mathrm{TSPP}_{\mathrm{r}} \mathrm{s}$ on other mutagens those listed in Fig. 1. As shown in Table 1, all the mutagens tested were changed to some extent after treatment with $\mathrm{Mn}$ -

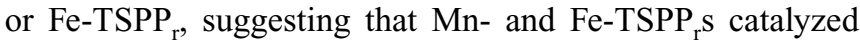
the oxidative reaction of respective mutagens with hydrogen peroxide under the conditions of recommended procedure described above. As can be seen from Table 1, IQ seems to be most oxidized among the HCAs tested by referring to $A_{\text {aq }}$, $A_{\text {sum }}$ and $A_{\text {control }}$. We therefore investigated the methanol-eluates of IQ in detail.

Determination of Optimal Condition The results described above suggest that $\mathrm{Mn}$ - and $\mathrm{Fe}-\mathrm{TSPP}_{\mathrm{r}} \mathrm{s}$ catalyze an oxidative reaction of IQ with hydrogen peroxide. The optimal reaction conditions were examined on the basis of UV/V spectra. The examinations were conducted using $1.0 \mathrm{ml}$ of $0.5 \mathrm{mmol} / \mathrm{IQ}, 1.0 \mathrm{ml}$ of hydrogen peroxide and $8.0 \mathrm{ml}$ of buffer solutions. In this case, the amount of IQ was four-fold to that of M-TSPP modified on IRA 900. Unfortunately, since M-TSPP ${ }_{r}$ is a solid, the number of turn over was not calculated.

(1) Effect of pH: As can be seen from Fig. 6, in the pH region tested $(\mathrm{pH} 4.0-11.0)$, the minimum absorbance at $263 \mathrm{~nm}$ was observed in the aqueous mixture after incubating at around $\mathrm{pH}$ 7.0, suggesting that IQ was oxidized by hydrogen peroxide in the presence of Mn- or Fe-TSPP ${ }_{r}$. In addition, the absorbance of methanol-eluate from $\mathrm{Mn}$ - or Fe$\mathrm{TSPP}_{\mathrm{r}}$ at $500 \mathrm{~nm}$, at which wavelength an oxidation product of IQ has a $\lambda_{\max }$, reached maximum between $\mathrm{pH} 7.0$ and 9.0. Thus, we selected $\mathrm{pH} 7.0$ as the optimal $\mathrm{pH}$.

(2) Effect of Hydrogen Peroxide: The UV/V spectra of the reaction mixtures and methanol-eluates were measured after treating IQ with Fe-TSPP ${ }_{\mathrm{r}}$ and hydrogen peroxide $(1.0 \mathrm{ml})$ of a different concentration between 0 and $100 \mathrm{mmol} / \mathrm{l}$. As the results, the absorbances around $263 \mathrm{~nm}$ of both the reaction

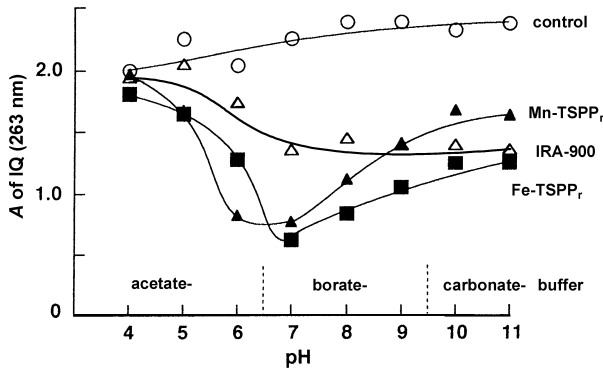

Fig. 6. Effect of $\mathrm{pH}$ to the Absorbance of IQ at $263 \mathrm{~nm}$ of the Reaction Mixture after Incubation

mixture and the methanol-eluate showed the minimum value when the concentration of hydrogen peroxide is around 5$10 \mathrm{mmol} / \mathrm{l}$. Thus, we decided to use $10 \mathrm{mmol} / \mathrm{l}$ as the optimal concentration of hydrogen peroxide solution $(1.0 \mathrm{ml})$.

(3) Effect of Incubation Temperature: The effect of incubation temperature was examined between 20 and $50^{\circ} \mathrm{C}$ on the basis of UV/V spectra of a reaction mixture and methanoleluate. As a result, only a small effect was observed. Thus, we selected $35^{\circ} \mathrm{C}$ as the optimal incubation temperature.

(4) Effect of Amount of Mn- or Fe-TSPP: The activities of the resins modified with Fe- or Mn-TSPP in the various rations from $5 \mu \mathrm{mol} / \mathrm{g}$ to $100 \mu \mathrm{mol} / \mathrm{g}(250-25 \mathrm{mg})$ were examined. As a result, the maximum absorbance of the methanol-eluate at $500 \mathrm{~nm}$, which is due to an oxidation product of IQ, was observed in the case where about $50 \mu \mathrm{mol}$ Fe- or Mn-TSPP/g was used. Thus, we selected $50 \mu \mathrm{mol} / \mathrm{g}$ as the optimal condition.

(5) Time Course: Figures 7a and b show the time courses of the absorbance at $263 \mathrm{~nm}$ of the reaction mixture, and the absorbance at $500 \mathrm{~nm}$ of the methanol-eluate, respectively. As can be seen from Fig. 7a, the absorbance decreased along with the incubation time until $120 \mathrm{~min}$, which means that the longer incubation time results in more adsorption and/or oxidation of IQ within said period of time. It is of particular interest on the progress of the oxidation reaction of IQ that absorbances are less in the cases of Fe- and Mn-TSPP ${ }_{r}$ compared to those in the case of IRA 900 . When $50 \mathrm{mg}$ of Mnor Fe-TSPP ${ }_{r}$ was used, the absorbance at $500 \mathrm{~nm}$ of the methanol-eluates increased as the incubation time became longer (see, Fig. 7b), suggesting that the oxidation product increased along with incubation time. In particular, Fe-TSPP generated an oxidation product more rapidly and rendered 

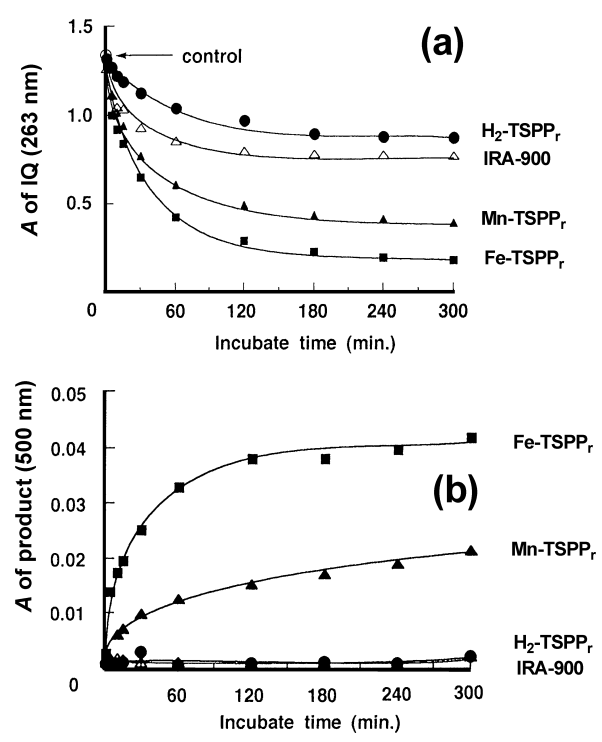

Fig. 7. Time Course of the Absorbance of IQ at $263 \mathrm{~nm}$ of the Reaction Mixture (a) and the Absorbance of the Oxidation Product at $500 \mathrm{~nm}$ in the Methanol-Eluates (b)

twice amount of product as compared to Mn-TSPP. It should be noted that the " $A$ "-value was closed to zero at $500 \mathrm{~nm}$ for non-modified IRA 900 (see, Fig. 7b), which indicates that $\mathrm{Mn}$ - and Fe-TSPP on IRA 900 catalyze oxidative reaction of IQ with hydrogen peroxide. Putting together Figs. 7a and b, the oxidative reaction of IQ seemed to be close to equilibrium at $120 \mathrm{~min}$. We then studied the methanol-eluate from Fe-TSPP $_{\mathrm{r}}$ after 120 min-incubation in detail to identify the oxidation product of IQ.

Identification of an Oxidation Product of IQ Figures $8 \mathrm{a}, \mathrm{b}$, and $\mathrm{c}$ show the chromatograms and UV/V spectra obtained from IQ solution, methanol-eluate from Fe-TSPP , and a solution of an oxidation product obtained by using horse radish POD, respectively. As can be seen from Fig. 8b, two major peaks, $\mathrm{B}$ and $\mathrm{C}$, were observed in chromatogram of the methanol-eluate. Since the UV/V spectrum of the peak B agreed with that of the peak A due to IQ (see, Fig. 8a), we identified the peak $B$ as IQ. The retention time of the peak $C$ coincided with that of the peak $\mathrm{D}$ due to the oxidation product. Furthermore, the spectrum of the peak $\mathrm{C}$ agreed with that of peak D as shown in Figs. 8b and c. These results strongly indicate that the oxidation product contained in the methanol-eluate is the same as that obtained by using horse radish $\mathrm{POD}$, which in term indicates that Fe-TSPP $\mathrm{r}_{\mathrm{r}}$ exhibits the POD-like catalytic activity for mutagens such as IQ.

To study the structure of the oxidation product, we measured the FAB-Mass and the ${ }^{1} \mathrm{H}-\mathrm{NMR}$ spectra. The peak observed at $199 \mathrm{~m} / \mathrm{e}$ can be assigned to $\left[\mathrm{IQ}+\mathrm{H}^{+}\right]$and the peak at $395 \mathrm{~m} / \mathrm{e}$ to [the oxidation product $+\mathrm{H}^{+}$]. The molecular weight (394) of the oxidation product is equal to $(2 \mathrm{IQ}-2 \mathrm{H})$, and the structure thereof in a methanol-eluate was estimated to be a dimer of IQ (hydorazone type, see Fig. 9) taking the $\mathrm{UV} / \mathrm{V}$ and ${ }^{1} \mathrm{H}-\mathrm{NMR}$ spectral data into consideration.

On the other hand, the data of the UV/V, the FAB-MS, the ${ }^{1} \mathrm{H}-\mathrm{NMR}$ spectra and the HPLC were obtained for the synthesized oxidation product. These data coincided with those of the oxidation product (the dimmer) separated from the methanol-eluate or the oxidation product obtained using

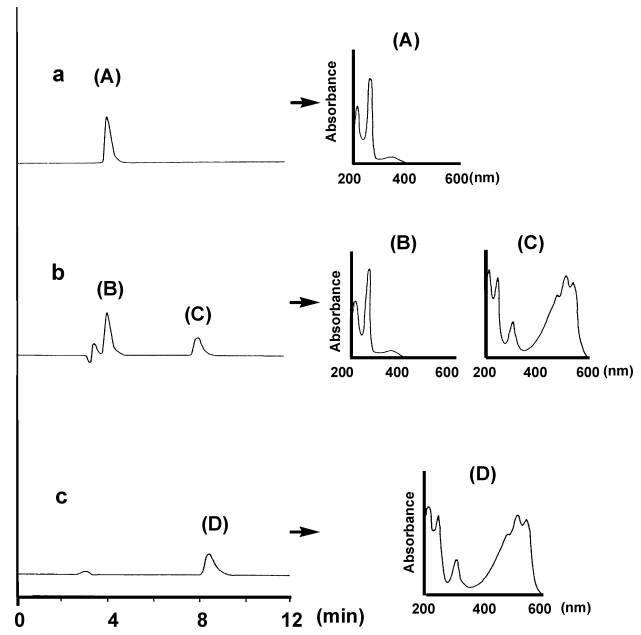

Fig. 8. Chromatograms and UV/V Spectra of the IQ Solution (a), Methanol-Eluate from Fe-TSPPr (b) and POD-Catalyzed Oxidation Product Solution (c)

Column, ODS (C-18) column; mobile phase, $80 \%$ methanol; flow rate, $0.5 \mathrm{~min} / \mathrm{min}$; detector, photodiode array detector.

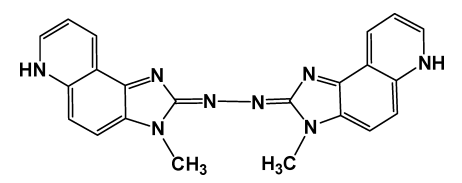

Fig. 9. Structure of IQ-Dimmer (Hydrazone Type)

horse radish POD. We therefore used the synthesized product in the investigation bellow.

Mutagenicity of IQ-Dimmer To examine the effect of dimerization on mutagenicity of IQ, umu-test ${ }^{14,15)}$ was carried out for IQ and its synthetic dimmer. As well known, IQ exhibits mutagenicity to Salmonella typhimurium TA98 at a low concentration such as $0.03 \mu \mathrm{g} / \mathrm{ml}$. However, the dimmer showed no mutagenicity to Salmonella typhimurium TA98 in the presence and the absence of S9 mix even at a high concentration such as $1.0 \mu \mathrm{g} / \mathrm{ml}$.

\section{Conclusion}

We revealed that some M-TSPP $\mathrm{s}$ exhibit a similar catalytic activity for HCAs to horse radish POD. In particular, Fe- and Mn-TSPP ${ }_{r}$ c catalyze the oxidation of IQ with hydrogen peroxide to give IQ-dimmer that has no mutagenicity in umu-test. Thus, we concluded that an M-TSPP ${ }_{\mathrm{r}}$ has a possibility to decrease mutagenicity of HCAs in vitro.

\section{References}

1) Skog K. I., Jahansson M. A. E., Jaegerstad M. I., Food Chem. Toxicol., 36, 32-48 (1998).

2) Hayatsu H., J. Chromatogr., A597, 37-56 (1992).

3) Skog K., J. Chromatogr, B802, 39-44 (2004).

4) Iwado H., Koyano M., Goto S., Kira S., Hayatsu H., Mut. Res., 322, 329-339 (1994).

5) Das C., Mishra H. N., Food Chemsitry, 68, 309-313 (2000).

6) Kasai H., Nakayama M., Yamazaki Z., Saito H., Nishihara H., Mut. Res., 164, 270-371 (1986).

7) Iwado I., Mifune M., Hazawa T., Mukuno T., Oda J., Motohashi N., Saito Y., Anal. Sci., 15, 841-846 (1999).

8) Mifune M., Iwado A., Akizawa H., Sugimoto K., Saito Y., Anal. Sci., 16, $1121-1125$ (2000).

9) Pasternack R. F., Francesconi L., Rhadd D., Spiro E., Inorg. Chem., 12, 2606-2611 (1973). 
10) Harriman A., Porter G., J. Chem. Soc., Faraday Trans., II, 75, 15321542 (1979).

11) Saito Y., Mifune M., Nakashima S., Tanaka Y., Chem. Pharm. Bull., 34, 5016-5019 (1986).

12) Hayatsu H., Ohara Y., Hayatsu T., Arimoto S., Saito Y., Mifune M., Tanaka Y., Mut. Res., 182, 384-385 (1987).
13) Ohara Y., Hayatsu T., Saito Y., Mifune M., Tanaka Y., Muraoka N., Hayatsu H., Mut. Res., 203, 384-385 (1988).

14) Oda Y., Nakamura S., Oki I., Kato T., Shinagawa H., Mut. Res., 147, 219-229 (1985)

15) Reifferscheid G., Heil J., Mut. Res., 369, 229-245 (1996). 